\title{
Measuring antigen-specific responses in Mycobacterium bovis-infected warthogs (Phacochoerus africanus) using the intradermal tuberculin test
}

Eduard O. Roos ${ }^{1}$, Francisco Olea-Popelka², Peter Buss³ ${ }^{3}$ Guy A. Hausler ${ }^{1}$, Robin Warren ${ }^{1}$, Paul D. van Helden ${ }^{1}$, Sven D. C. Parsons ${ }^{1}$, Lin-Mari de Klerk-Lorist ${ }^{4}$ and Michele A. Miller ${ }^{1 *}$

\begin{abstract}
Background: Bovine tuberculosis (bTB) caused by Mycobacterium bovis has previously been diagnosed in warthogs and infection can be highly prevalent (>30\%) in endemic areas. Thus, warthogs could potentially be an important species to consider as sentinels for disease surveillance. However, disease surveillance is dependent on availability of accurate diagnostic assays and only a few diagnostic tests have been investigated for warthogs. Furthermore, the tests that have been used in this species require laboratory equipment and trained personnel to obtain results. Therefore, this study investigated the use of the intradermal tuberculin test (ITT) to screen warthogs for bTB, which can be done with minimal equipment and under field conditions by most veterinarians and other qualified professionals. Changes in skin fold thickness measurements at the bovine purified protein derivative (PPD) administration site, between 0 and $72 \mathrm{~h}$, were compared with differential changes between the bovine and avian PPD sites, for 34 warthogs, to evaluate the performance when different interpretation criteria for the ITT was used.

Results: Using an increase of $1.8 \mathrm{~mm}$ or more at the bovine PPD site as a cut-off for positive responders, $69 \%$ of 16 M. bovis culture-positive warthogs had a positive test result, with $100 \%$ of the 18 culture-negative warthogs considered as test negative. When a differential of $1.2 \mathrm{~mm}$ or more in skin fold thickness at the bovine PPD compared to the avian PPD site was used as a cut-off for the comparative ITT, $81 \%$ of culture-positive warthogs were considered as test positive, with 100\% of culture-negative warthogs considered as test negative.
\end{abstract}

Conclusion: The findings in this study suggest that the ITT is a promising tool to use when screening warthogs for M. bovis infection.

Keywords: Bovine tuberculosis, Screening test, Mycobacterium bovis, Intradermal tuberculin test, Warthog, Wildlife

\section{Background}

The primary cause of bovine tuberculosis (bTB) is an acid-fast bacterium, Mycobacterium bovis, which has been reported to infect more than 17 wildlife species in South Africa [1]. The disease has become endemic in some nature reserves and private game farms within South Africa [2]. Furthermore, cases of bTB have been

\footnotetext{
* Correspondence: miller@sun.ac.za

${ }^{1}$ NRF-DST Centre of Excellence for Biomedical Tuberculosis Research, South African Medical Research Council Centre for Tuberculosis Research, Division of Molecular Biology and Human Genetics, Faculty of Medicine and Health Sciences, Stellenbosch University, PO Box 241, Cape Town 8000, South Africa Full list of author information is available at the end of the article
}

reported in threatened or endangered species, such as lions (Panthera leo) and rhinoceros (Ceratotherium simum and Diceros bicornis) [3, 4]. Certain wildlife species have become maintenance hosts of the disease in South Africa including the African buffalo (Syncerus caffer) [5]. Warthogs are also known to become infected with $M$. bovis and could potentially act as a maintenance host in endemic areas $[6,7]$. This species is capable of crossing fences and other man-made barriers, which could lead to dissemination of disease, as is the case for wild boar (Sus scrofa), a bTB maintenance host in the Iberian Mediterranean ecosystem [1, 8]. Moreover,

C The Author(s). 2018 Open Access This article is distributed under the terms of the Creative Commons Attribution 4.0 International License (http://creativecommons.org/licenses/by/4.0/), which permits unrestricted use, distribution, and 
similar to feral pigs, warthogs may serve as a good sentinel as they are highly susceptible to this infection [9].

Accurate diagnostic tests are needed for disease surveillance. However, only a limited number of assays are available for bTB diagnosis in African wildlife species. A lack of approved laboratory facilities and logistical difficulties in getting samples to laboratories from disease controlled and remote areas also limits wildlife testing. One available method for field detection of bTB is to euthanase animals, identify granulomatous lesions on necropsy, and confirm the diagnosis using mycobacterial culture. However, it can take 6-8 weeks before culture results become available. Therefore, there is a need to have an accurate field-friendly ante-mortem assay for bTB screening of species such as warthogs, which can be readily performed by veterinarians.

The Intradermal Tuberculin Test (ITT) has been used for bTB detection in a range of species including domestic cattle, wild boar, white-tailed deer (Odocoileus virginianus), elk (Cervus canadensis), African buffalo, and lions [10-15]. The ITT is readily available to veterinarians and can be performed in the field, providing a result within $72 \mathrm{~h}$. The objective of this study was to investigate the utility and test performance of the ITT for detection of $M$. bovis infection in warthogs.

\section{Results}

The ITT was measured for all 34 warthogs and individuals divided into two study cohorts based on mycobacterial culture results (Table 1). M. bovis-infection was confirmed by mycobacterial culture in 16 of the 34 warthogs. The SFT measurements from warthogs infected with NTMs were not significantly different from that of $M$. bovis culture-negative warthogs, for both PPD sites, and therefore grouped as culture-negative $(p=0.086)$. There was no significant difference in $\triangle \mathrm{PPD}_{\mathrm{a}}$ measurements between $M$. bovis culture-positive and culture-negative warthogs ( $p=0.650$, Additional file 1: Figure S1, Table 2). However, $\triangle \mathrm{PPD}_{\mathrm{b}}$ measurements were significantly greater for culture-positive compared to culture-negative animals (Fig. 1, Table 2). Furthermore, in $M$. bovis culture-positive warthogs, the increase in SFT at the $\mathrm{PPD}_{\mathrm{b}}$ site $\left(\triangle \mathrm{PPD}_{\mathrm{b}}\right)$ was significantly greater than at the $\mathrm{PPD}_{\mathrm{a}}$ site $\left(\Delta \mathrm{PPD}_{\mathrm{a}}\right)(p$ $=0.002$ ), although no differences between these measurements were seen in culture-negative warthogs $(p=0.128$, Additional file 1: Figure S1, Table 2). The $\mathrm{PPD}_{\mathrm{b}-\mathrm{a}}$ values were significantly greater for culture-positive warthogs compared to culture-negative warthogs $(p<0.0001$, Fig. 2, Table 2).

A warthog-specific cut-off value for the SITT was calculated as $\geq 1.8 \mathrm{~mm}$ using a ROC curve analysis (AUC = 0.91, 95\% CI 0.81-1.0) (Additional file 2: Table S1). Based on this cut-off, 11 out of the $16 \mathrm{M}$. bovis culture-positive warthogs were SITT-positive (69\%), while none of the 18 culture-negative warthogs had a positive test result (100\%).

The cut-off value for the CITT was $\geq 1.2 \mathrm{~mm}$ (AUC = 0.91, 95\% CI 0.79-1.0) (Additional file 3: Table S2). This cut-off value resulted in 13 of 16 culture-positive warthogs being CITT-positive (81\%) and classified all 18 culture-negative warthogs as test negative (100\%). No signs of oedema, heat, exudation or necrosis were observed at the PPD injection sites in any of the 34 warthogs tested.

\section{Discussion}

This study shows that the ITT could distinguish between $M$. bovis culture-positive and negative warthogs from bTB endemic regions of South Africa, using both the SITT and CITT interpretations. Warthog specific cut-off values for the SITT and CITT were calculated to be $\geq 1.8 \mathrm{~mm}$ and $\geq 1.2 \mathrm{~mm}$, respectively. The optimal ITT criterion for detection of infected warthogs in this study was the $\geq 1.2 \mathrm{~mm}$ cut-off for the CITT, which resulted in correct classification of $81 \%$ of culture-positive animals as CITT-positive and $100 \%$ of culture-negative warthogs as test negative. These results suggest that the interpretation of the ITT in this species should include the reaction to avian PPD (i.e., CITT) to identify the highest number of infected animals.

Importantly, diagnostic application in each species requires optimization and standardization of the ITT, as the injection site and dose may influence the delayed-type hypersensitivity response, with variable interpretation affecting test sensitivity $[11,12,14,16]$. In this study, a double dose of tuberculin ( $0.2 \mathrm{ml}$ PPD) was injected intradermally, caudal to each ear in warthogs, to minimise the chance of delayed-type hypersensitivity response failure due to dose, as is the case in lions and domestic cats [11].

The choice of ITT (SITT or CITT) is dependent on the prevalence and exposure of $M$. bovis as well as the presence of sensitising NTMs in a population [10]. The SITT is a simpler test, since it consists of one injection and measurement, although it lacks the discriminatory power of the CITT, where the response to $\mathrm{PPD}_{\mathrm{a}}$ identifies sensitisation to NTMs [10]. In this study, the response at the $\mathrm{PPD}_{\mathrm{a}}$ site was significantly less than that at the $\mathrm{PPD}_{\mathrm{b}}$ site, indicating that the increase in SFT at the $\mathrm{PPD}_{\mathrm{b}}$ site was a true measure of $M$. bovis infection and not a cross-reactive response to NTMs.

In many countries the initial recommendations that were in place for the ITT suggested the use of the single intradermal cervical test as the primary bTB screening test and the CITT as an ancillary test [10]. However, most of these countries have amended their regulations, as the CITT has been shown to be a more specific test than the SITT [17]. Furthermore, our results showed 
Table 1 Raw data from 34 warthogs' skinfold measurements (in $\mathrm{mm}$ ) to $\mathrm{PPD}_{\mathrm{b}}$ and $\mathrm{PPD}_{\mathrm{a}}$ at time points $0 \mathrm{~h}$ and $72 \mathrm{~h}$ post-injection, the delta $\mathrm{PPD}_{\mathrm{b}}$ and $\mathrm{PPD}_{\mathrm{a}}$ as well as each warthogs' mycobacterial culture result

\begin{tabular}{|c|c|c|c|c|c|c|c|}
\hline Lab no. & $\mathrm{PPD}_{\mathrm{b}} \mathrm{Oh}$ & $\mathrm{PPD}_{\mathrm{b}} 72 \mathrm{~h}$ & $\triangle P P D_{b}$ & $\mathrm{PPD}_{\mathrm{a}} \mathrm{Oh}$ & $\mathrm{PPD}_{\mathrm{a}} 72 \mathrm{~h}$ & $\triangle P P D_{a}$ & Bacterial Culture \\
\hline $15 / 137$ & 3 & 3 & 0 & 3.1 & 3.1 & 0 & Culture negative \\
\hline $15 / 138$ & 3.6 & 4.1 & 0.5 & 3.6 & 4.8 & 1.2 & Culture negative \\
\hline $15 / 262$ & 4.2 & 3.8 & -0.4 & 4.6 & 5 & 0.4 & Culture negative \\
\hline $15 / 271$ & 4.9 & 4.9 & 0 & 4.3 & 4.8 & 0.5 & Culture negative \\
\hline $15 / 302$ & 2.6 & 4 & 1.4 & 2.7 & 4.6 & 1.9 & Culture negative \\
\hline $15 / 304$ & 3 & 3 & 0 & 3 & 3.3 & 0.3 & Culture negative \\
\hline $15 / 307$ & 5 & 6.1 & 1.1 & 4 & 6.6 & 2.6 & Culture negative \\
\hline $15 / 308$ & 3.3 & 3.3 & 0 & 3.7 & 3.8 & 0.1 & Culture negative \\
\hline $15 / 310$ & 3 & 3.2 & 0.2 & 3 & 3.4 & 0.4 & Culture negative \\
\hline $15 / 305$ & 3.2 & 4.2 & 1 & 2.8 & 3.1 & 0.3 & M. asiaticum \\
\hline $15 / 516$ & 5.3 & 4.2 & -1.1 & 5.5 & 4.7 & -0.8 & M. asiaticum \\
\hline $15 / 309$ & 3.2 & 3.6 & 0.4 & 3.3 & 3.4 & 0.1 & M. avium \\
\hline $15 / 301$ & 2.3 & 2.4 & 0.1 & 2.6 & 2.5 & -0.1 & M. intracellulare \\
\hline $15 / 515$ & 3.9 & 3.4 & -0.5 & 3.3 & 3 & -0.3 & M. intracellulare \\
\hline $15 / 535$ & 3.8 & 3.8 & 0 & 5.5 & 5.2 & -0.3 & M. paraffinicum \\
\hline $15 / 514$ & 4.8 & 4.7 & -0.1 & 4 & 5.2 & 1.2 & M. simiae \\
\hline $15 / 534$ & 4.6 & 5.3 & 0.7 & 4.8 & 4.2 & -0.6 & M. simiae \\
\hline $15 / 536$ & 4.5 & 4.5 & 0 & 4.5 & 5 & 0.5 & M. simiae \\
\hline $15 / 140$ & 4.8 & 6.9 & 2.1 & 4.6 & 4.6 & 0 & M. bovis \\
\hline $15 / 248$ & 3.8 & 8 & 4.2 & 4 & 3.8 & -0.2 & M. bovis \\
\hline $15 / 249$ & 4.4 & 4.4 & 0 & 4.5 & 4.7 & 0.2 & M. bovis \\
\hline $15 / 250$ & 3.6 & 7 & 3.4 & 4.3 & 4.1 & -0.2 & M. bovis \\
\hline $15 / 251$ & 4.8 & 5.7 & 0.9 & 4.1 & 4 & -0.1 & M. bovis \\
\hline $15 / 263$ & 4.1 & 7.2 & 3.1 & 3.9 & 3.8 & -0.1 & M. bovis \\
\hline $15 / 264$ & 3.8 & 14 & 10.2 & 3.2 & 3.4 & 0.2 & M. bovis \\
\hline $15 / 265$ & 4.3 & 4.9 & 0.6 & 3.8 & 3.8 & 0 & M. bovis \\
\hline $15 / 266$ & 4.3 & 11.2 & 6.9 & 4.2 & 3.6 & -0.6 & M. bovis \\
\hline $15 / 267$ & 3.8 & 6.5 & 2.7 & 4.4 & 4.6 & 0.2 & M. bovis \\
\hline $15 / 268$ & 3.6 & 6.4 & 2.8 & 3.6 & 4.1 & 0.5 & M. bovis \\
\hline $15 / 269$ & 3.7 & 4.7 & 1 & 2.9 & 3.5 & 0.6 & M. bovis \\
\hline $15 / 270$ & 2.8 & 5.9 & 3.1 & 2.9 & 3.2 & 0.3 & M. bovis \\
\hline $15 / 300$ & 3.6 & 7 & 3.4 & 3.6 & 4 & 0.4 & M. bovis \\
\hline $15 / 306$ & 3.3 & 8.1 & 4.8 & 3.8 & 4.6 & 0.8 & M. bovis \\
\hline $15 / 513$ & 4.8 & 5.1 & 0.3 & 4.6 & 6.2 & 1.6 & M. bovis \\
\hline
\end{tabular}

Table 2 Median values of the skinfold increase (in $\mathrm{mm}$ ) using different combinations of measurements for the ITT in $M$. bovis culture-positive and culture-negative warthogs

\begin{tabular}{lll}
\hline Skinfold reading & Culture Positive & Culture Negative \\
\hline$\triangle P P D_{\mathrm{a}}$ & $0.2 \mathrm{~mm}(-0.1-0.5)$ & $0.3 \mathrm{~mm}(-0.2-0.7)$ \\
$\triangle P D_{\mathrm{b}}$ & $3.0 \mathrm{~mm}(0.9-4.0)$ & $0.0 \mathrm{~mm}(-0.1-0.6)$ \\
$\mathrm{PPD}_{\mathrm{b}}-\mathrm{PPD}_{\mathrm{a}}$ & $2.5 \mathrm{~mm}(1.3-3.5)$ & $-0.4 \mathrm{~mm}(-0.5-0.1)$
\end{tabular}

Interquartile ranges are shown in parentheses that the CITT was more sensitive than the SITT $(81 \%$ versus 69\%, correctly identifying animals with a culture-positive result). This may be due to the more sensitive cut-off value of $\geq 1.2 \mathrm{~mm}$ for the CITT compared to the $\geq 1.8 \mathrm{~mm}$ cut-off for the SITT $[10,18,19]$. It is important to note that neither the CITT nor SITT had any false positives in this study using these criteria.

Unfortunately, no biological test is perfect (i.e. has $100 \%$ sensitivity and specificity) and the CITT could not correctly classify all $M$. bovis culture-positive warthogs 

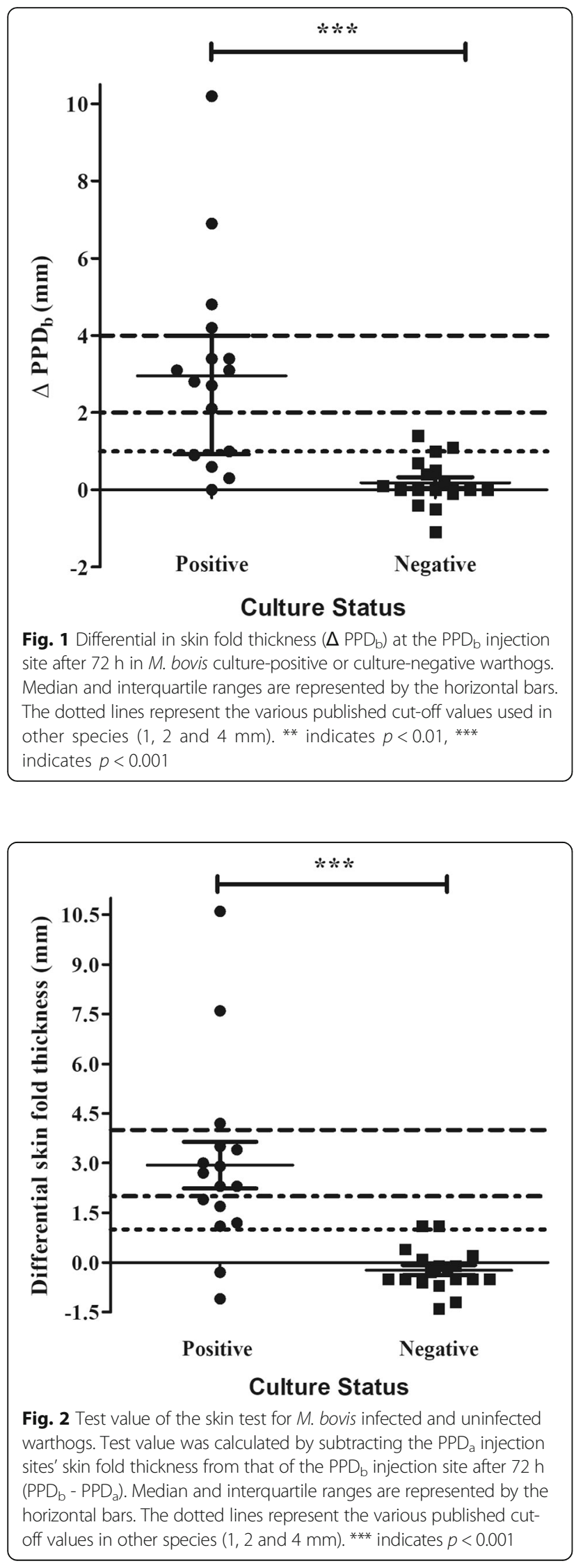

as test positive and at $81 \%$, it is comparable to the sensitivity of the CITT in cattle [20]. The sub-optimal sensitivity could be due to various factors, for example, anergy, co-infection with NTMs, immunosuppression associated with nutritional, immobilization or transport stress, operator error, faulty equipment, or tuberculin not correctly administered intradermally [10].

For disease surveillance, the cut-off value of a screening test should be set to optimize sensitivity and specificity, after considering the prevalence of disease and epidemiological factors as well as clinical and financial constraints $[19,21]$. A cost-effective and logistically feasible method is required for disease surveillance in wildlife since access to laboratories may be limited. Although previous reports have shown that serological assays can be used to identify infected warthogs [6], these require laboratory equipment and techniques which may not be readily available. Therefore, the ITT may be an alternative screening test to laboratory-based assays in some situations.

One limitation of this study was that cut-off values and test specificity were determined using endemic controls rather than animals from a known $M$. bovis negative population. Therefore, future research should ideally include an unexposed population of warthogs to evaluate specificity and determine a diagnostic cut-off value for the ITT.

\section{Conclusion}

This study demonstrates that an antigen-specific in vivo response to $M$. bovis can be measured in warthogs when challenged with $\mathrm{PPD}_{\mathrm{b}}$, thus confirming the usefulness of the ITT for this species. Cut-off values determined by ROC curve analyses were able to distinguish between $M$. bovis culture-positive and culture-negative warthogs with good sensitivity and specificity. Interpretation of the ITT under the criteria followed for the CITT, allowed greater numbers of infected warthogs to be detected. Thus, the application of the ITT will be a valuable tool for disease surveillance in warthogs.

\section{Methods}

Animals and sampling

In 2015, warthogs were captured and culled as part of drought management in the Greater Kruger National Park area (GKNP) by park veterinarians [22]. Since bTB is endemic in the GKNP, all warthogs in this study were considered exposed to $M$. bovis. Sixteen female and eighteen male warthogs were first immobilized and held in quarantine bomas to evaluate performance of the ITT in this species, as described below. Based on physical examination at the time of capture and post-mortem evaluation, all the animals were deemed to be healthy and in good to moderate body condition. The authors did not find evidence of any condition that would result 
in an immunocompromised animal, when the ITT was read. After reading the ITT, immobilized warthogs were humanely euthanized using succinylcholine $(25 \mathrm{mg} / \mathrm{kg}$; Kyron Laboratories, Benrose, South Africa), saturated with potassium chloride, administered intravenous. This drug was chosen since the warthog was already unconscious, the drug was readily available, could be administered in sufficient quantities to result in rapid death to a large animal, and did not present a health hazard if meat was consumed (by scavengers or others). Since the warthogs were part of a disease surveillance program, all animals were euthanized and a full necropsy performed. Post-mortem examination and tissue sampling were performed as previously described [6]. In summary, lymph nodes were collected from all warthogs and examined for gross lesions consistent with bTB. If no visible legions were observed, samples were pooled according to anatomical site and all sets cultured.

\section{Immobilization}

All warthogs were immobilized using a drug combination of (i) zolazepam-tiletamine (Zoletil ${ }^{\circledR}$; Virbac RSA, (Pty) Ltd., Centurion, South Africa) in combination with azaperone (Kyron Laboratories (Pty) Limited, Benrose, South Africa) or medetomidine (Kyron), or (ii) azaperone, butorphanol (Kyron), medetomidine and ketamine (Kyron) [22-24]. Immobilizations and holding conditions complied with the South African National Parks Standard Operating Procedures for the Capture, Transportation and Maintenance in Holding Facilities of Wildlife.

\section{Intradermal tuberculin test (ITT)}

The intradermal tuberculin test was performed as described elsewhere [11]. Briefly, the skin fold thickness (SFT) caudal to each ear was measured using a spring loaded Hauptner calliper with pistol grip prior to administration of the purified protein derivative (PPD; Institute for Animal Sciences, Lelystadt, Netherlands) injection (Additional file 4: Figure $\mathrm{S} 2)$. PPD was injected intradermally at $0 \mathrm{~h}: 0.2 \mathrm{ml}$ bovine PPD $(30,000 \mathrm{IU} / \mathrm{ml})\left(\mathrm{PPD}_{\mathrm{b}}\right)$ on the left and $0.2 \mathrm{ml}$ avian $\mathrm{PPD}(25,000 \mathrm{IU} / \mathrm{ml})\left(\mathrm{PPD}_{\mathrm{a}}\right)$ on the right. After $72 \mathrm{~h}$, the SFT at each PPD injection site was measured and examined for signs of oedema, heat, exudation or necrosis [25]. The same experienced operator performed all measurements and PPD injections.

The ITT was interpreted in two ways. For the single intradermal tuberculin test (SITT), the SFT prior to the $\mathrm{PPD}_{\mathrm{b}}$ injection $(0 \mathrm{~h})$ was subtracted from the measurement at the same site $72 \mathrm{~h}$ post-injection $\left(\triangle \mathrm{PPD}_{\mathrm{b}}\right)$. For the comparative intradermal tuberculin test (CITT), the $72 \mathrm{~h}$ SFT at the $\mathrm{PPD}_{\mathrm{a}}$ injection site was subtracted from that at the $\mathrm{PPD}_{\mathrm{b}}$ injection site $\left(\mathrm{PPD}_{\mathrm{b}-\mathrm{a}}\right)$. This was done since the degree of dehydration varies between individuals. Thus, only the absolute increase in response was used to calculate the $\mathrm{PPD}_{\mathrm{b}}$ specific response. The degree of dehydration in individual animals has also been recognized by the South African Department of Agriculture, Forestry and Fisheries in TB testing of African buffaloes [26]. The $\triangle \mathrm{PPD}_{\mathrm{a}}$ was calculated in the same way as the $\triangle \mathrm{PPD}_{\mathrm{b}}$.

\section{Mycobacterial cultures and speciation}

All tissue samples were processed using the BACTEC ${ }^{\mathrm{Tm}}$ MGIT $^{\mathrm{TM}} 960$ system (BD Biosciences, New Jersey, USA) as previously described [13]. Positive cultures in the MGIT system were further analysed by Ziehl-Neelsen (ZN) staining and all $\mathrm{ZN}$-positive cultures were speciated using genetic region of difference analysis [27] and 16S DNA sequencing [28]. The status of warthogs as $M$. bovis-infected or uninfected was based on culture results.

\section{Data analysis}

Statistical analyses were performed using GraphPad Prism version 5 (GraphPad Software, March 2007). The $\triangle \mathrm{PPD}_{\mathrm{b}}$ and $\triangle \mathrm{PPD}_{\mathrm{a}}$ values for $M$. bovis culture-positive and culture-negative animals were compared within and between groups using a Kruskal-Wallis statistic with a Dunn's Multiple Comparison test. The $\Delta \mathrm{PPD}_{\mathrm{a}}$ and $\Delta$ $\mathrm{PPD}_{\mathrm{b}}$ comparisons were done to confirm the specific response of $M$. bovis culture-positive warthogs to the $\mathrm{PPD}_{\mathrm{b}}$ injection. Evaluation of the $\triangle \mathrm{PPD}_{\mathrm{a}}$ was done to provide information as to whether non-tuberculous mycobacteria (NTM) influence the $\mathrm{PPD}_{\mathrm{b}}$ responses. The SITT results for culture-positive and culture-negative animals were compared using a Mann Whitney test, as were results for the CITT. Warthog specific cut-off values to determine positive responders were calculated using a receiver operator characteristic (ROC) curve analysis and selected based on the Youden's index [29].

\section{Additional files}

Additional file 1: Figure S1. Differential in skin fold thickness at the $P P D_{b}$ and $P P D_{a}\left(\triangle P P D_{b}\right.$ and $\left.\triangle P P D_{a}\right)$ injection site after $72 \mathrm{~h}$ in $M$. bovis culture-positive $(C P)$ or culture-negative $(C N)$ warthogs. Median and interquartile ranges are represented by the horizontal bars. ${ }^{* *}$ indicates $p<0.01,{ }^{* * *}$ indicates $p<0.001$. (TIF $614 \mathrm{~kb}$ )

Additional file 2: Table S1. Receiver operator characteristics curve analysis data for $\triangle P P D_{b}$. Warthog specific cut-off values and respective sensitivity and specificity, with $95 \% \mathrm{Cl}$ in parentheses. Youden's index for each cut-off value is also indicated. (DOCX $15 \mathrm{~kb}$ )

Additional file 3: Table S2. Receiver operator characteristics curve analysis data for $\mathrm{PPD}_{\mathrm{b}}-\mathrm{PPD}_{\mathrm{a}}$. Warthog specific cut-off values and respective sensitivity and specificity, with $95 \% \mathrm{Cl}$ in parentheses. Youden's index for each cut-off value is also indicated. (DOCX 14 kb)

Additional file 4: Figure S2. Purified protein derivative injection site for the intradermal tuberculin test, caudal to each ear. The picture shows the appropriate use of the callipers to measure the skin fold thickness ( $\mathrm{mm})$. (PNG $1964 \mathrm{~kb}$ ) 


\section{Acknowledgements}

The authors wish to acknowledge and thank the following individuals for their contributions to this study: Leana Rossouw, Tebogo Manamela, Dr. Jennie Hewlett, Marius Kruger and the Veterinary Wildlife Services capture team from Kruger National Park for assistance with sample collection from the warthogs, as well as Schalk van Dyk, At Dekker, Rudi Lorist and the state veterinary Quarantine Boma Team for sample collection and general care of warthogs being housed inside the quarantine facility.

\section{Funding}

South African Medical Research Council and the National Research Foundation of South Africa [SARChl grant 86949]. The content is the sole responsibility of the authors and does not necessarily represent the official views of the funders.

\section{Availability of data and materials}

All data generated or analysed during this study are included in this published article and its supplementary information files.

\section{Authors' contributions}

EOR, L-MdK-L, SDCP and MAM conceptualised and designed the project. EOR, L-MdK-L, MAM, PB, GAH were all part of sampling and sample processing. EOR and FO-P were responsible for the data analysis and interpretation. SDCP, L-MdK-L and MAM supervised the findings and encourage EOR to investigate these. FO-P, PB, PDvH and RW supported the research with critical revision of the article. EOR, SDCP, L-MdK-L and MAM drafted the article with all authors contributed substantially to the writing and revising of the manuscript. All authors approved the final version for submission (EOR, FO-P, PDvH, GAH, RW, PDvH, SDCP, L-MdK-L and MAM).

\section{Ethics approval}

This study received ethical clearance from the Stellenbosch University Animal Care and Use committee (SU-ACUD15-00029).

\section{Consent for publication}

Not applicable.

\section{Competing interests}

The authors declare that they have no competing interests.

\section{Publisher's Note}

Springer Nature remains neutral with regard to jurisdictional claims in published maps and institutional affiliations.

\section{Author details}

${ }^{1}$ NRF-DST Centre of Excellence for Biomedical Tuberculosis Research, South African Medical Research Council Centre for Tuberculosis Research, Division of Molecular Biology and Human Genetics, Faculty of Medicine and Health Sciences, Stellenbosch University, PO Box 241, Cape Town 8000, South Africa. ${ }^{2}$ Department of Clinical Sciences, College of Veterinary Medicine and Biomedical Sciences, Colorado State University, 300 W. Drake Rd, Fort Collins, CO 80523, USA. ${ }^{3}$ Veterinary Wildlife Services, South African National Parks, Kruger National Park, Private Bag X402, Skukuza 1350, South Africa. ${ }^{4}$ Office of the State Veterinarian, Kruger National Park, Department of Agriculture, Forestries and Fisheries, PO Box 12, Skukuza 1350, South Africa.

\section{Received: 24 May 2018 Accepted: 2 November 2018}

\section{Published online: 20 November 2018}

\section{References}

1. Michel AL, Bengis RG, Keet DF, Hofmeyr M, De Klerk LM, Cross PC, et al. Wildlife tuberculosis in South African conservation areas: Implications and challenges. Vet Microbiol. 2006;112:91-100. Available from: http://www. sciencedirect.com/science/article/pii/S0378113505003834. [cited 19 Jan 2015].

2. Hlokwe TM, van Helden P, Michel AL. Evidence of increasing intra and interspecies transmission of Mycobacterium bovis in South Africa: Are we losing the battle? Prev Vet Med. 2014;115:10-7. https://doi.org/10.1016/j. prevetmed.2014.03.011.

3. Sylvester TT, Martin LER, Buss P, Loxton AG, Hausler GA, Rossouw L, et al. Prevalence and risk factors for Mycobacterium bovis infection in African lions
(Panthera leo) in the Kruger National Park. J Wildl Dis. 2017;53:372-6 Available from: http://www.jwildlifedis.org/doi/10.7589/2016-07-159.

4. Miller MA, Buss PE, van Helden PD, Parsons SDC. Mycobacterium bovis in a free-ranging black rhinoceros, Kruger National Park, South Africa, 2016. Emerg Infect Dis. 2017;23:557-8.

5. De Vos V, Bengis RG, Kriek NP, Michel A, Keet DF, Raath JP, et al. The epidemiology of tuberculosis in free-ranging African buffalo (Syncerus caffer) in the Kruger National Park, South Africa. Onderstepoort J Vet Res. 2001;68: $119-30$.

6. Roos EO, Buss P, de Klerk-Lorist L-M, Hewlett J, Hausler GA, Rossouw L, et al. Test performance of three serological assays for the detection of Mycobacterium bovis infection in common warthogs (Phacochoerus africanus). Vet Immunol Immunopathol. 2016;182:79-84. https://doi.org/10. 1016/j.vetimm.2016.10.006.

7. Renwick $A R$, White $P C L$, Bengis $R G$. Bovine tuberculosis in southern African wildlife: a multi-species host-pathogen system. Epidemiol Infect. 2007;135:529. Available from: http://www.pubmedcentral.nih.gov/ articlerender.fcgi?artid $=2870607 \&$ tool $=$ pmcentrez\&rendertype $=$ abstract. [cited 19 Jan 2015].

8. Naranjo V, Gortazar C, Vicente J, de la Fuente J. Evidence of the role of European wild boar as a reservoir of Mycobacterium tuberculosis complex. Vet Microbiol. 2008;127:1-9. Available from: http://www.sciencedirect.com/ science/article/pii/S0378113507004853. [cited 23 Feb 2015].

9. Roos EO, Olea-Popelka F, Buss P, de Klerk-Lorist L-M, Cooper D, van Helden PD, et al. Seroprevalence of Mycobacterium bovis infection in warthogs (Phacochoerus africanus) in bovine tuberculosis-endemic regions of South Africa. Transbound Emerg Dis. 2018;65:1182-9. Available from: http://doi. wiley.com/10.1111/tbed.12856.

10. de la Rua-Domenech R, Goodchild AT, Vordermeier HM, Hewinson RG, Christiansen KH, Clifton-Hadley RS. Ante mortem diagnosis of tuberculosis in cattle: A review of the tuberculin tests, gamma-interferon assay and other ancillary diagnostic techniques. Res Vet Sci. 2006;81:190-210. Available from: http://www.sciencedirect.com/science/article/pii/S0034528806000026. [cited 16 Jan 2015].

11. Keet DF, Michel AL, Bengis RG, Becker P, van Dyk DS, van Vuuren M, et al. Intradermal tuberculin testing of wild African lions (Panthera leo) naturally exposed to infection with Mycobacterium bovis. Vet Microbiol. 2010;144:38491. https://doi.org/10.1016/j.vetmic.2010.01.028.

12. Jaroso R, Vicente J, Fernandez-de-Mera IG, Aranaz A, Gortazar C. Eurasian wild boar response to skin-testing with mycobacterial and nonmycobacterial antigens. Prev Vet Med. 2010;96:211-7. https://doi.org/10. 1016/j.prevetmed.2010.06.011.

13. Goosen WJ, Miller MA, Chegou NN, Cooper D, Warren RM, van Helden PD, et al. Agreement between assays of cell-mediated immunity utilizing Mycobacterium bovis-specific antigens for the diagnosis of tuberculosis in African buffaloes (Syncerus caffer). Vet Immunol Immunopathol. 2014;160: 133-8. Available from: http://www.sciencedirect.com/science/article/pii/ S0165242714000816. [cited 5 Jan 2015].

14. Palmer MV, Whipple DL, Waters WR. Tuberculin skin testing in white-tailed deer (Odocoileus virginianus). J Vet Diagn Investig. 2001;13:530-3 Available from: http://www.ncbi.nlm.nih.gov/pubmed/11724147.

15. Palmer MV, Whipple DL, Payeur JB, Bolin CA. Use of the intradermal tuberculin test in a herd of captive elk (Cervus elaphus nelsoni) naturally infected with Mycobacterium bovis. J Vet Diagn Investig. 2011;23:363-6.

16. Good M, Clegg TA, Costello E, More SJ. The comparative performance of the single intradermal test and the single intradermal comparative tuberculin test in Irish cattle, using tuberculin PPD combinations of differing potencies. Vet J. 2011;190:e60-5. Available from: http://linkinghub.elsevier. com/retrieve/pii/S1090023311000128. [cited 13 June 2016].

17. Goodchild AV, Downs SH, Upton P, Wood JLNN, De La Rua-Domenech R, De Rua-domenech R. Specificity of the comparative skin test for bovine tuberculosis in Great Britain. Vet Rec. 2015;177:258.

18. Metz CE. Basic principles of ROC analysis. Semin Nucl Med. 1978;8:283-98 Available from: http://www.sciencedirect.com/science/article/pii/ S0001299878800142.

19. Greiner M, Pfeiffer D, Smith RD. Principles and practical application of the receiver-operating characteristic analysis for diagnostic tests. Prev Vet Med. 2000;45:23-41.

20. Hartnack S, Torgerson PR. The Accuracy of the single intradermal comparative skin test for the diagnosis of bovine Tuberculosis-estimated from a systematic literature search. Mycobact Dis. 2012;2. Available from: 
https://www.omicsonline.org/the-accuracy-of-the-single-intradermalcomparative-skin-test-for-the-diagnosis-of-bovine-tuberculosis-estimatedfrom-a-systematic-literature-search-2161-1068.1000120.php?aid=8272. Accessed 13 June 2016.

21. van Erkel AR, Pattynama PM. Receiver operating characteristic (ROC) analysis: basic principles and applications in radiology. Eur J Radiol. 1998;27:88-94.

22. SANParks. Coordinated Policy Framework Governing Park Management Plans [Internet]. SANParks Manag. Plan Policy Framew; 2006. p. 60. Available from: https://www.sanparks.org/docs/conservation/cpfjanuary2010.pdf.

23. Hewlett J. Evaluation of a partially reversible immobilization protocol using Medetomidine, Butorphanol, Zolazepam-Tiletamine, and ketamine in freeranging warthogs (Phacochoeus africanus) in Kruger National Park. University of Pretoria; 2017.

24. Moon PF, Smith L. General anesthetic techniques in swine. Vet Clin North Am Food Anim Pract. 1996;12:663-91. Available from: http://www.ncbi.nlm. nih.gov/pubmed/8916392.

25. OIE WO for AH. OIE. OIE Terr. Man. 2009 [Internet]. Paris; 2009. p. 1-16. Available from: http:/www.oie.int/fileadmin/Home/eng/Healthstandards/ tahm/2.04.07BOVINETB.pdf. Accessed 13 June 2016.

26. DAFF D of AF and F. Buffalo disease risk management [Internet]. South Africa; 2017. p. 1-15. Available from: https://www.daff.gov.za/vetweb/ pamphlets\&Information/Policy/Buffalo\%20Disease\%20Risk\%20 Management\%20VPN\%20\%20ADDENDUMS_2017-02-17.pdf. Accessed 13 June 2016.

27. Warren RM, Gey van Pittius NC, Barnard M, Hesseling A, Engelke E, de Kock $M$, et al. Differentiation of Mycobacterium tuberculosis complex by PCR amplification of genomic regions of difference. Int J Tuberc Lung Dis. 2006; 10:818-22.

28. Leclerc M-C, Haddad N, Moreau R, Thorel M-F. Molecular characterization of environmental Mycobacterium strains by PCR-restriction fragment length polymorphism of hsp65 and by sequencing of hsp65, and of 165 and ITS1 rDNA. Res Microbiol. 2000;151:629-38. Available from: http://www. sciencedirect.com/science/article/pii/S0923250800901293. [cited 8 July 2015].

29. Youden WJ. Index for rating diagnostic tests. Cancer. 1950;3:32-5.

Ready to submit your research? Choose BMC and benefit from:

- fast, convenient online submission

- thorough peer review by experienced researchers in your field

- rapid publication on acceptance

- support for research data, including large and complex data types

- gold Open Access which fosters wider collaboration and increased citations

- maximum visibility for your research: over $100 \mathrm{M}$ website views per year

At $\mathrm{BMC}$, research is always in progress.

Learn more biomedcentral.com/submissions 